\title{
INFLUÊNCIA DE EXERCÍCIOS ATIVOS LIVRES E DE ALTO IMPACTO NO FORTALECIMENTO DA MUSCULATURA PÉLVICA
}

Giuliano Roberto da SILVA ${ }^{1}$

Gerusa Dias Siqueira Vilela TERRA ${ }^{2}$

Cassiano Merussi NEIVA ${ }^{3}$

Marcelo Rodrigo TAVARES ${ }^{4}$

César Augusto Costa RODRIGUES ${ }^{5}$

Yvan Fernandes VILAS BOAS ${ }^{6}$

Cíntia Paula de Lima DIAS ${ }^{7}$

\begin{abstract}
${ }^{1}$ Graduação Licenciatura Plena em Educação Física pela FAGAMMON - Lavras - MG
Especialização em Ginástica Especial Corretiva - UNIFMU - SP

Especialização em Diversidade e Gênero na Escola - UFLA - Lavras - MG

Mestrado em Biotecnologia em Saúde - UNINCOR - Três Corações - MG

Doutorando em Promoção de Saúde na Universidade de Franca - UNIFRAN - Franca - SP

Docente na Faculdade Presbiteriana Gammon - FAGAMMON - Lavras - MG - Brasil

Docente na Universidade José do Rosário Vellano - UNIFENAS - Alfenas - MG - Brasil

Docente no Centro Universitário do Sul de Minas Gerais - UNISMG - Varginha - MG - Brasil

Docente na Secretaria Regional de Ensino de Minas Gerais - SER - Varginha - MG - Brasil

E-mail: giumusc@gmail.com
\end{abstract}

${ }^{2}$ Graduação em Fisioterapia pela UNIFENAS - Alfenas - MG

Mestrado em Saúde - UNIFENAS - Alfenas - MG

Doutoranda em Promoção de Saúde na Universidade de Franca - UNIFRAN - Franca - SP

Docente na Universidade José do Rosário Vellano - UNIFENAS - Alfenas - MG - Brasil

E-mail: gerusa.terra@unifenas.br

${ }^{3}$ Graduação em Educação Física pela UNESP - SP

Mestrado em Ciências da Motricidade Humana pela UNESP - SP

Doutorado em Biologia Funcional e Molecular (Fisiologia e Bioquímica) pela UNICAMP - Campinas - SP

Pós Doutorado em Bioquímica (Metabolismo e Bioenergética) pela UNESP - SP

Pós Doutorado em Child Physiology Exercise pela Charles University - CUNI

Pós Doutorado em Bioquímica y Fisiolgia del Esfuerzo pela Universidad Politécnica de Madrid - UPM - Espanha

Pós Doutorado em Nuevas Abordajes en Fisiología, Nutrición y Bioquimica del Esfuerzo pela Universidad Politécnica de Madrid - UPM- Espanha

Livre Docente - LD no conjunto de disciplinas: Nutrição Humana e Metabolismo, Bioquímica e Fisiologia do Esforço e Antropometria pela Universidade Estadual Paulista Júlio de Mesquita Filho - UNESP - SP

Docente na Universidade Estadual Paulista Júlio de Mesquita Filho - UNESP - SP - Brasil

Docente na Universidad Politécnica de Madrid - UPM - Espanha

Docente e orientador no Programa de Mestrado e Doutorado em Promoção de Saúde na Universidade de Franca UNIFRAN - SP - Brasil

Docente na Universidade José do Rosário Vellano - UNIFENAS - MG - Brasil

Docente na Universidade de Ribeirão Preto - UNAERP - SP - Brasil

E-mail: cassiano.neiva@unifenas.com

${ }^{4}$ Graduação em Fisioterapia pela UNIFENAS - Alfenas - MG

Mestrado em Bioengenharia pela Universidade de São Paulo - USP - SP

Doutorado em Medicina (Neurologia) pela Universidade de São Paulo - USP -SP

Docente na Universidade José do Rosário Vellano - UNIFENAS - Alfenas - MG - Brasil

E -mail: marcelo1tavares@yahoo.com.br 
${ }^{5}$ Graduação em Educação Física pela UNINCOR -Três Corações - MG

Mestrado em Saúde Coletiva pela UNIVÁS - Pouso Alegre - MG

Docente na Universidade José do Rosário Vellano - UNIFENAS - Alfenas - MG - Brasil

E-mail: cesarsgs@ hotmail.com

${ }^{6}$ Graduação em Educação Física pela UFSJ - Juiz de Fora - MG

Especialização em Fisiologia do Exercício e Avaliação Morfo-Funcion pela Universidade Gama Filho (UGF) - RJ

Docente na Universidade José do Rosário Vellano - UNIFENAS - Alfenas - MG - Brasil

e-mail: yvanvilasboas@yahoo.com.br

${ }^{7}$ Discente no curso de Educação Física na Universidade José do Rosário Vellano - UNIFENAS - Alfenas - MG -

Brasil.

E-mail: cintiapaulaedf@hotmail.com

\title{
Recebido em: 24/08/2015 - Aprovado em: 06/01/2016 - Disponibilizado em: 30/07/2016
}

\begin{abstract}
RESUMO
O objetivo do trabalho foi avaliar o efeito de exercícios livres na musculatura do assoalho pélvico, associados a exercícios de alto impacto como Jump e Step. O estudo foi realizado com 60 mulheres, de 20 a 65 anos, onde inicialmente foram submetidas a um teste de força da musculatura do assoalho pélvico, através do método bidigital, e após, foram realizadas aulas de Jump e Step associadas a exercícios livres na musculatura pélvica, durante 8 semanas, em 3 sessões de 60 minutos, em dias alternados. Ao final do treinamento, as mesmas foram submetidas novamente ao mesmo teste, sendo os resultados classificados de acordo com a Escala de Ortiz. Percebeu-se que após as 8 semanas de exercícios, $20 \%$ da amostra foi classificada com grau 0; $20 \%$ grau 2; e $60 \%$ grau 3. O fortalecimento da musculatura pélvica associada a exercícios de impacto foi eficiente em um programa de exercícios livres, considerando $\mathrm{p} \leq 0,05$.

Palavras-chave: Fortalecimento da musculatura pélvica. Incontinência urinária. Exercícios livres. Exercícios de alto impacto.
\end{abstract}

\begin{abstract}
The objective was to evaluate the effect of free exercises on the pelvic floor muscles associated with high-impact exercises such as Jump and Step. The study was conducted with 60 women, 20 to 65, which were initially subjected to a test of strength of the pelvic floor muscles through bidigital method, and after, were carried Jump and Step classes associated with free exercises in the musculature pelvic for 8 weeks, 3 sessions of 60 minutes every other day. At the end of the training, they were again subjected to the same test and the results were ranked according to Scale Ortiz. It was noticed that after 8 weeks of exercise, $20 \%$ of the sample was classified as grade $0 ; 20 \%$ grade 2 ; and $60 \%$ grade 3 . Strengthening the pelvic muscles associated with impact exercises was effective in a free exercise program, considering $\mathrm{p} \leq 0.05$.
\end{abstract}

Keywords: Strengthening the pelvic muscles. Urinary incontinence. Free exercises. High impact exercises.

\section{INTRODUÇÃO}

Os exercícios moderados sempre foram recomendados por contribuírem e aprimorarem a saúde, porém, há evidências consistentes e recentes de que os exercícios de alta intensidade ou vigorosos também produzem efeitos significativos e importantes para a saúde e proporcionam maior dispêndio energético diário (BATISTA et al., 2003). Os exercícios mais intensos contribuem também de forma positiva para a saúde, particularmente aqueles relacionados ao aumento do gasto energético, aumento da massa corporal magra, aumento do dispêndio de energia pós-exercício, redução do perfil lipídico, influenciando reduções de até duas vezes as taxas de mortalidade (FURTADO, SIMÕES \&LEMOS, 2004).

Porém, em contrapartida, algumas literaturas mostram que nos exercícios físicos de alto impacto ocorre o enfraquecimento do assoalho pélvico causando a Incontinência Urinária em mulheres, e para esse tipo de 
exercícios, como o jump e o step estudados nesse trabalho, essa patologia é comumente presente (ALMEIDA \& MACHADO, 2012).

A Incontinência Urinária é definida pela Sociedade Internacional de Continência como sendo "a perda involuntária de urina, que determina desconforto social e higiênico, podendo ser demonstrável de forma objetiva" (ABRAMS et al., 2003). É um sintoma comum que afeta mulheres de todas as idades, podendo interferir seriamente no bem-estar físico, psicológico e social dos indivíduos afetados (RETT, 2007).

As perdas urinárias ocorrem quando há um aumento da pressão intra-abdominal por esforços, tais como tosse, espirro, risada, saltar, caminhar ou orgasmo. A forma de incontinência urinária que apresenta maior prevalência é a incontinência urinária de esforço genuína, sendo responsável por $60 \%$ de todos os casos de incontinência urinária feminina (BERNARDES et al., 2000).

A prevalência da Incontinência Urinária aumenta com a idade e ocorre também em mulheres jovens e nulíparas (LOPES \& HIGA, 2006). Nestas mulheres, o exercício extenuante resulta em menor pressão de contração vaginal, uma vez que há um comprometimento do suprimento sanguíneo e debilidade de oxigênio para as fibras musculares do tipo I, responsáveis pela manutenção do tônus, indicando fadiga muscular do pavimento pélvico. Além disso, existe uma redução do sinal neural após atividade muscular prolongada, reduzindo a eficiência da contração muscular e favorecendo a perda da continência (BERNARDES et al., 2000).

Quando a Incontinência Urinária é estudada em mulheres praticantes de exercícios físicos, diversos estudos referem que sua fisiopatologia esteja relacionada às pressões constantes sobre o assoalho pélvico e aumentos abruptos da pressão abdominal que ocorrem durante a prática de atividades físicas. Mesmo em mulheres com os músculos do assoalho pélvico fortes, a atividade física árdua levaria ao aumento da pressão abdominal, aliada à sobrecarga, ao estiramento e ao enfraquecimento do assoalho pélvico, acarretando em Incontinência Urinária (FOZZATTI et al., 2008; ARAÚJO et al., 2008).

Movimentos de alto impacto resultam em força de impacto sobre o assoalho pélvico três a quatro vezes maiores que o peso corporal (KNORST, 2012). Mais especificamente relata-se que a força vertical de reação máxima do solo durante diferentes atividades esportivas é três a quatro vezes o peso do corpo quando corre, cinco a doze vezes quando pula, e nove vezes na queda após um salto em altura. Em mulheres que praticam exercícios físicos com maior frequência semanal a prevalência de Incontinência Urinária também é mais frequente (ELIASSON, LARSSON \& MATTSSON, 2002). 
É importante ressaltar também que a busca por uma vida sexual mais prazerosa tem sido imprescindível, além de ser um indicador de qualidade de vida. Quanto mais forte a musculatura do assoalho pélvico estiver, maior será o prazer durante a relação sexual. Para que isso ocorra, se faz necessário uma carga maior de exercícios específicos para o assoalho pélvico, visto que, além de fortalecer, proporciona à mulher maior coordenação motora dos músculos, a partir da qual, diferentes manobras podem incrementar e desenvolver a relação (RACCO, 2006).

Visto que o profissional educador físico tem de estar atento para a sinergia dos exercícios e do corpo, e que os benefícios da prática de exercícios são inquestionáveis, se torna necessário um plano de exercícios de fortalecimento do assoalho pélvico juntamente aos exercícios de maior impacto.

Diante da simplicidade do exercício para fortalecimento pélvico e a sua relevância, sendo usado não somente na prevenção e fortalecimento, mas muitas vezes até no tratamento da incontinência e disfunções sexuais causadas pelo enfraquecimento pélvico, viu-se a possibilidade de um estudo abrangendo exercícios de alto impacto como o jump e o step associados a exercícios específicos para fortalecimento pélvico com análise do aumento de força muscular do assoalho pélvico e melhoria da qualidade de vida, para a mulher não deixar de praticar seus exercícios normalmente (ALMEIDA \& MACHADO, 2012).

Logo, o objetivo do estudo foi mensurar o grau de força da musculatura do assoalho pélvico, avaliando o efeito de exercícios ativos livres nesta musculatura, quando associados a exercícios de impacto.

\section{MÉTODO}

O estudo foi realizado em uma academia exclusiva para mulheres localizada no município de Alfenas/MG. As voluntárias foram esclarecidas sobre o estudo e, em seguida, assinaram um Termo de Consentimento Livre e Esclarecido (TCLE), de acordo com a Resolução no 196/96 do Conselho Nacional de Saúde.

Participaram 60 mulheres, com idade entre 20 e 65 anos, saudáveis, alunas das práticas de Jump e Step na academia supracitada, que não realizavam nenhum outro exercício além deste. As voluntárias, inicialmente foram submetidas a uma avaliação de força da musculatura do assoalho pélvico através do teste bidigital aplicado por uma fisioterapeuta. Neste teste, as mulheres foram inspecionadas em posição ginecológica, seguindo todos os padrões de assepsia. Foi observada, através da palpação, durante a contração muscular ativa: simetria, trofismo, força e contração reflexa da musculatura do assoalho pélvico em repouso e durante a tosse. Foi observado também se houve sensibilidade e dor. Foi aplicado também, um questionário estruturado 
para caracterização da amostra.

Após avaliação inicial as voluntárias foram submetidas a 8 semanas consecutivas de treinamento, 3 vezes por semana. Cada sessão teve duração de 1 hora e consistiu de 10 minutos de aquecimento, seguido de 15 minutos de Jump e 15 minutos de Step. Após, foi feito um trabalho específico para fortalecimento da musculatura pélvica com exercícios ativos livres, com duração de 20 minutos. Ao final da aula foi estabelecido 10 minutos de alongamento e relaxamento, respectivamente. Após estas 8 semanas de treinamento, as voluntárias foram reavaliadas pela mesma fisioterapeuta.

Os exercícios realizados com o objetivo de fortalecimento da musculatura pélvica utilizados no estudo foram extraídos do livro de Silva (SILVA, 2011).

A análise estatística dos dados neste estudo foi realizada através do teste t de Student, considerando um nível de significância de $95 \%$, ou seja, $\mathrm{p} \leq 0,05$.

\section{Escala de Ortiz}

O grau de força muscular foi classificado de acordo com a Escala de Ortiz (ORTIZ, NUNEZ \& IBANEZ, 1996) (Tabela1).
Tabela 1 - Grau de força muscular do assoalho pélvico Grau de Força Muscular do Assoalho Pélvico

\begin{tabular}{|ll|}
\hline Grau 0 & $\begin{array}{l}\text { Sem função perineal objetiva, nem mesmo } \\
\text { à palpação; }\end{array}$ \\
Grau 1 & $\begin{array}{l}\text { Função perineal objetiva ausente, } \\
\text { reconhecida somente à palpação; }\end{array}$ \\
Grau 3 & $\begin{array}{l}\text { Função perineal objetiva débil, } \\
\text { reconhecida à palpação; }\end{array}$ \\
Função perineal objetiva e resistência \\
opositora, não mantida à palpação; 4 \\
$\begin{array}{l}\text { Função perineal objetiva e resistência } \\
\text { opositora, mantida à palpação por mais de } \\
\text { cinco segundos. }\end{array}$ \\
\hline
\end{tabular}

Fonte: Ortiz, Nunez e Ibanez (1996)

\section{Exercícios de Alto Impacto}

Jump Fit: é um programa de exercícios ritmados sobre um minitrampolim, sendo seus benefícios considerados os mesmos que os alcançados pela prática regular dos exercícios aeróbios. O sucesso deste programa está relacionado, principalmente, ao prazer e motivação que esta atividade proporciona, além da obtenção ou manutenção dos níveis de adequados de condicionamento físico para a realização das tarefas do cotidiano (SIMÃO, 2004).

Step Training: é uma atividade aeróbia de intensidade fisiológica baixa ou moderada e de longa duração. Utiliza como recurso uma plataforma regulável em altura, sobre a qual são efetuados movimentos em diferentes planos. A altura da plataforma varia entre 10 $\mathrm{cm}$ e $30 \mathrm{~cm}$ aproximadamente, e a movimentação pode ou não ser coreografada, mas é sempre cadenciada por música (JUCÁ, 1993). Para Olson, Williford e Blessing (1991), e Martinovic, Marques e Novaes (2002), a demanda cardiorrespiratória desta atividade é adequada para melhoria aeróbia. 


\section{Exercícios Ativos Livres}

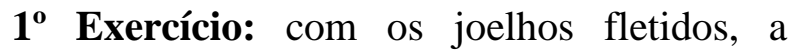
voluntária tentou realizar uma retroversão pélvica, ao mesmo tempo em que se solicita a atitude de contrair o períneo como se fosse interromper o jato de urina, mantendo-se de 4 a 5 segundos na posição e repetindo por oito vezes.

$2^{\circ}$ Exercício: em decúbito dorsal, com membros inferiores fletidos, pés posicionados e apoiados, membros superiores ao longo do corpo. A voluntária elevou o quadril, fazendo ponte, contraiu os glúteos por 10 segundos, relaxando por 10 segundos entre um movimento e outro, repetindo-o por 10 vezes.

$3^{\circ}$ Exercício: a voluntária em decúbito dorsal, com os membros inferiores fletidos, pés posicionados e apoiados, membros superiores ao longo do corpo, manteve-se a postura de contrair os joelhos contra a bola (que esta entre os joelhos) por 3 segundos, relaxando em seguida, repetindo 4 séries de 10 repetições.

$4^{0}$ Exercício: com a mesma postura do exercício anterior, entretanto com contração dos glúteos unificada à elevação do quadril. Foi realizado 4 séries de 10 repetições.

$5^{\circ}$ Exercício: a voluntária em decúbito ventral, com o quadril sobre um travesseiro, contraiu a musculatura glútea, mantendo por 3 segundos e relaxando-a em seguida. Foram realizadas 4 séries de 10 repetições.
$6^{0}$ Exercício: com o tronco inclinado para frente apoiando os antebraços nos joelhos, com abdução dos membros inferiores, contraindo os músculos da vagina como se fosse interromper o ato miccional mantendo cada contração até 5 segundos, depois relaxando. Foram realizadas 8 séries. Depois de cada contração, imaginar que esta urinando $\mathrm{e}$, de repente, interromper o jato miccional. $\mathrm{O}$ tempo de repouso entre as séries foram de 80 segundos. Após, foram realizadas 10 séries mantendo as contrações por 10 segundos. Nessa fase o tempo de relaxamento foi igual ao de contrações.

$7^{\circ}$ Exercício: ante e retroversão: a voluntária se senta ereta sobre a bola terapêutica, os membros inferiores ficam abduzidos e os pés pressionam firmemente o chão. Faz uma flexão do tronco; as mãos são posicionadas sobre as coxas para sustentar o peso do tronco e os cotovelos ficam flexionados durante o exercício. As tuberosidades do ísquio iniciam o movimento e rolam a bola para frente, fazendo uma retroversão de pelve, e para trás, fazendo a anteversão pélvica. Esses exercícios são para a consciência corporal e relaxamento do assoalho pélvico, além de fortalecimento pélvico.

$8^{\circ}$ Exercício: direita-esquerda: para limitar a flexão lateral do tronco, a voluntária levantou os braços no plano transverso da cintura escapular e do queixo, pressionando a mão firmemente, uma contra a outra. A coluna permanece em postura ereta e a voluntária 
continua a respirar normalmente, cuidando para que não ocorra a manobra de Valsalva. Inicia-se o movimento de rolamento para o lado oposto, da direita para o lado esquerdo, depois a tuberlosidade esquerda para o lado direito.

9 Exercício: a voluntária em bipedestação com joelhos fletidos. Foram feitos movimentos de anteversão e retroversão de quadril com 20 repetições.

$\mathbf{1 0}^{\circ}$ Exercício: a mesma posição do exercício anterior, com os membros superiores ao longo do corpo e relaxados, pés distantes a aproximadamente 20 centímetros um do outro, contraindo os glúteos o máximo que puder, mantendo por 20 segundos, realizando o movimento por 10 vezes.

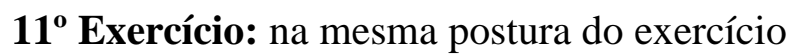
anterior, a voluntária flexionou os joelhos, com as mãos posicionadas na cintura e os pés paralelos a 20 centímetros um do outro, fazendo um movimento circular, imitando os movimentos de quando se usa um bambolê. São quatro movimentos que completam o exercício: primeiro, a retroversão; segundo, levando o quadril á esquerda; terceiro, realizou a anteversão; e, por último, o quadril vai para a direita. Foram realizados 10 giros com a retroversão da pelve, ao mesmo tempo em que foi contraída a musculatura do assoalho pélvico. Depois de fazer a anteversão da pelve, contraiu a musculatura do assoalho pélvico, e contou 3 segundos para cada posição, e em seguida, relaxou. Este movimento foi repetido por 10 vezes.

\section{RESULTADOS E DISCUSSÃO}

Os resultados obtidos através do questionário e da avaliação fisioterapêutica foram expressos em gráficos.

A amostra analisada apresentou o seguinte perfil: $40 \%$ das participantes praticavam atividade física regular a menos de 1 ano; $20 \%$ praticavam de 1 a 3 anos; e $40 \%$ praticavam a mais de 3 anos regularmente (Figura 1).

FIGURA 1 - Tempo de atividade física regular

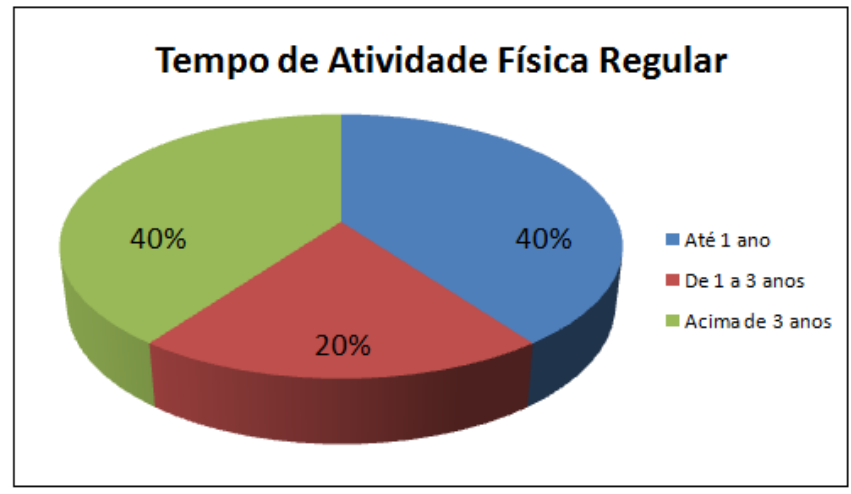

Fonte: Autores

Destas participantes que praticavam atividades físicas regularmente, $80 \%$ praticavam de forma específica Jump e Step, regularmente, a menos de 1 ano; 20\% Jump e Step de 1 a 3 anos, e nenhuma das integrantes praticavam Jump e Step a mais de 3 anos (Figura 2). 
FIGURA 2 - Tempo que praticam Jump e Step

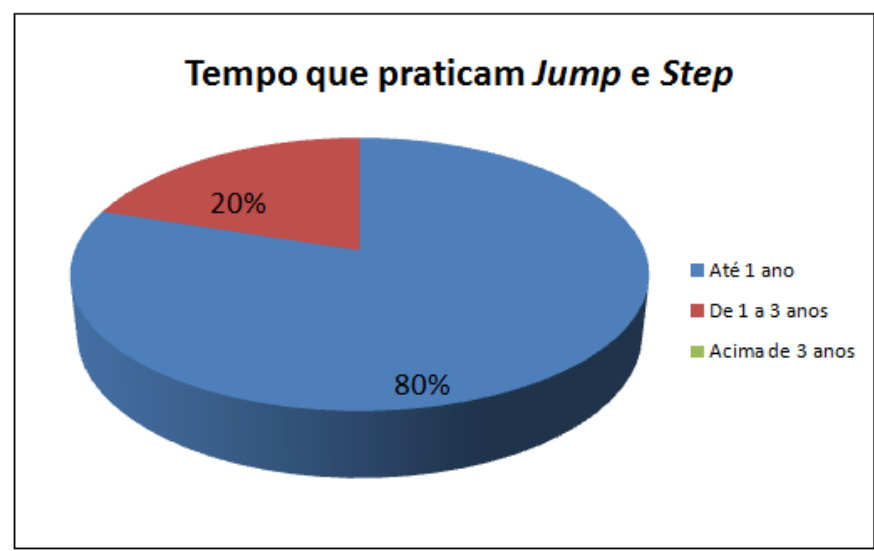

Fonte: Autores

A Figura 3 refere-se à vida sexual do grupo estudado, mostrando que $20 \%$ possuíam vida sexual inativa, $20 \%$ regular, $20 \%$ boa e $40 \%$ consideraram ótima a vida sexual.

FIGURA 3 - Classificação da vida sexual

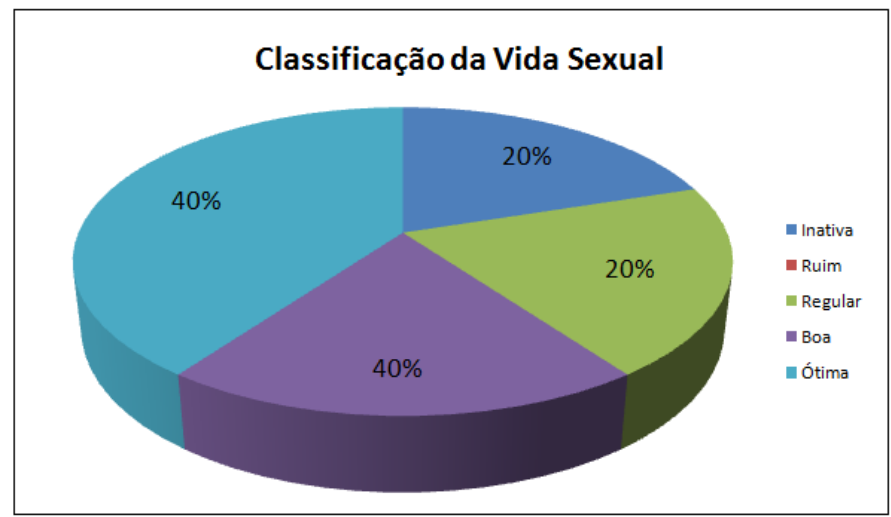

Fonte: Autores

Com relação ao fortalecimento da musculatura pélvica foi possível observar que houve uma evolução significante. Antes do início do trabalho, 20\% da amostra apresentava grau $0 ; 40 \%$ apresentava grau 1; e $40 \%$ grau 2. Após as oito semanas de exercícios, $20 \%$ da amostra continuava com grau $0(\mathrm{p}=0,05)$, enquanto que as participantes que apresentaram grau 1 (40\%) baixou para
$0 \% \quad(\mathrm{p}=0,04) ;$ as participantes que apresentaram grau 2, baixou de $40 \%$ para $20 \%(\mathrm{p}=0,032) ;$ e $60 \%$ das participantes alcançaram o grau $3(\mathrm{p}=0,38)$, que antes da realização dos exercícios, não foi alcançado por nenhuma das participantes. Este resultado confirma a eficiência dos exercícios perineais (Figura 4).

FIGURA 4 - Função da contração da musculatura do assoalho pélvico antes e depois

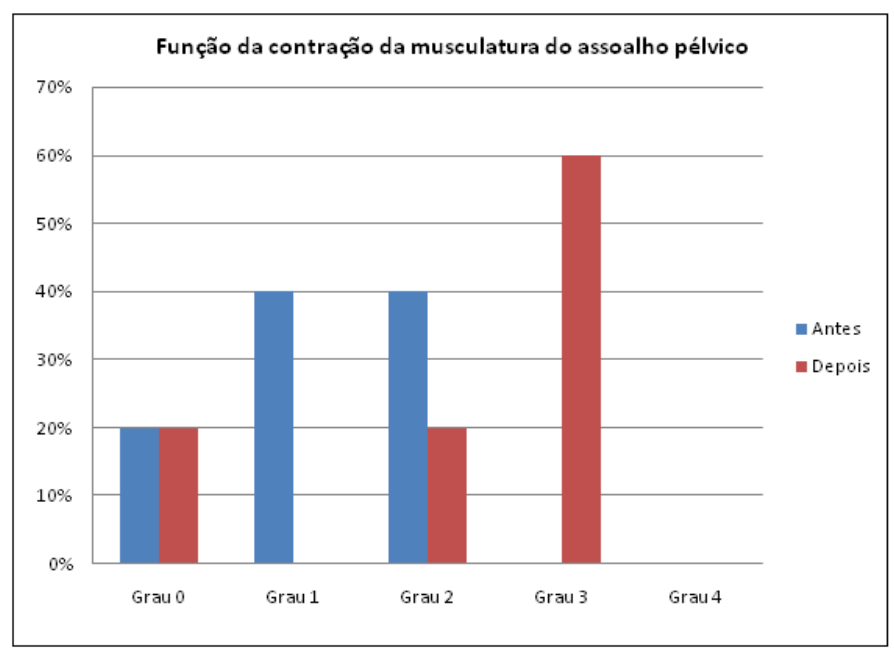

Fonte: Autores

No trabalho desenvolvido por Matheus, Mazzari e Mesquita (2006), foram avaliadas 12 mulheres apresentando queixa de incontinência urinária, submetidas a 10 sessões fisioterapêuticas, duas vezes na semana, divididas em dois grupos: Grupo A: $\mathrm{n}=6$, utilizando exercícios perineais e Grupo $B: \mathrm{n}=6$, utilizando cones vaginais, onde realizaram exercícios posturais para correção estática da pelve. Foram coletados os dados do grau de contração muscular do períneo antes e após intervenção pela Avaliação 
Funcional do Assoalho Pélvico sendo encontrados os seguintes dados (Figura 5).

FIGURA 5 - Valores médios do grau de força muscular do assoalho pélvico, pré e pós-intervenção (* $\mathrm{p} \leq 0,05)$

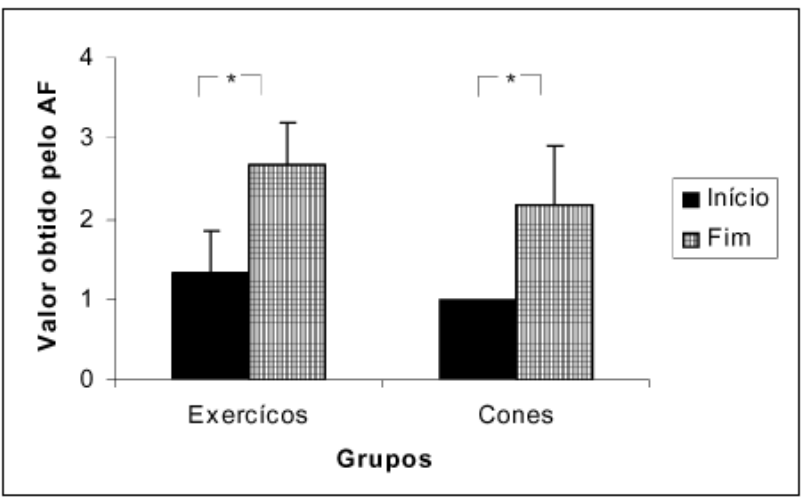

Fonte: Matheus, Mazzari e Mesquita (2006)

Quando comparados os níveis de contração da musculatura do assoalho pélvico, nota-se semelhança entre os resultados, pois em ambas as pesquisas, houve melhora significativa da função de contração pélvica. Porém na presente pesquisa houve adição de exercícios de alto impacto como Jump e Step. Mediante a esta análise pode-se afirmar que, exercícios de fortalecimento da musculatura pélvica associados a exercícios de alto impacto, como Jump e Step, não sofrem alterações quanto ao grau de contração da musculatura do assoalho pélvico.

De acordo com Simão (2004), exercícios de alto impacto induzem ao enfraquecimento da musculatura do assoalho pélvico devido ao aumento da pressão dos órgãos sobre as estruturas pélvicas. Contudo, contrariando os estudos deste autor, na presente pesquisa não houve comprovação da influência dos exercícios de alto impacto sobre o assoalho pélvico, quando estes vêm associados à contração voluntária da musculatura do assoalho pélvico.

\section{CONCLUSÃO}

Conclui-se que o programa de exercícios ativos livres realizados por 8 semanas foi eficiente para o fortalecimento da musculatura pélvica, mesmo associado a exercícios de alto impacto (Jump e Step).

\section{REFERÊNCIAS}

ABRAMS, P.; CARDOZO, L.; FALL, M.; GRIFFITHS, D.; ROSIER, P.; ULMSTEN, U.; VAN KERREBROECK, P.; VICTOR, A.; WEIN, A. Standardisation Sub-Committee of the International Continence Society. The standardisation of terminology of lower urinary tract function: report from the standardisation sub-committee of the International Continence Society. Urology, v.61, n.1, p. 37-49, 2003.

ALMEIDA, P.P.; MACHADO, L.R.G. A prevalência de incontinência urinária em mulheres praticantes de jump. Fisioter. mov., v.25, n.1, p. 61-70, 2012.

ARAÚJO, M.P.; OLIVEIRA, E.; ZUCCHI, E.V.M.; TREVISANI, V.F.M.; GIRÃO, M.J.B.C.; SARTORI, M.G.F. Relação entre incontinência urinária em mulheres atletas corredoras de longa distância e distúrbio alimentar. Rev. Assoc. Med. Bras., v.54, n.2, p.146-49, 2008.

BATISTA, D.C.; CHIARA, V.L.; GUGELMIN, S.A.; MARTINS, P.D.

Atividade física e gestação: saúde da gestante não atleta e crescimento fetal. Rev. Bras. 
Saude Mater. Infant., v. 3, n.2, p. 32-45, 2003.

BERNARDES, N.O.; PÉRES, F.R.; SOUZA, E.L.B.L.; SOUZA, O.L. Métodos de Tratamento Utilizados na Incontinência Urinária de Esforço Genuína: um Estudo Comparativo entre Cinesioterapia e Eletroestimulação Endovaginal. Rev. Bras.

Ginecol. Obstet., v.22, n.1, p. 56-61, 2000.

ELIASSON, K.; LARSSON, T.; MATTSSON, E. Prevalence of stress incontinence in nulliparous elite trampolinists. J. Med. Sci. Sports., v.12, n.2, p. 106-10, 2002.

FOZZATTI, M.C.M.; PALMA, P.; HERRMANN, V.; DAMBROS, M. Impacto da reeducação postural global no tratamento da incontinência urinária de esforço feminina. Rev. Assoc. Med. Bras., v.54, n.1, p. 17-22, 2008.

FURTADO, E.; SIMÃO, R.; LEMOS, A. Análise do consumo de oxigênio, freqüência cardíaca e dispêndio energético, durante as aulas do Jump Fit. Rev. Bras. Med., v.10, n.5, p. 109-123, 2004.

JUCÁ, M. Aeróbica e step: bases fisiológicas e metodológicas. Rio de Janeiro: Sprint, 1993.

KNORST, M.R.; CAVAZZOTTO, K.; HENRIQUE, M.; RESENDE, T.L.

Intervenção fisioterapêutica em mulheres com incontinência urinária associada ao prolapso de órgão pélvico. Rev. Bras. Fisioter., v.16, n.2, p. 187-199, 2012.

LOPES, M.H.B.M.; HIGA, R. Restrições causadas pela incontinência urinária à vida da mulher. Ver. Esc. Enferm., v.40, n.1, p. 3941, 2006.

MARTINOVIC, N.V.P.; MARQUES, M.B.; NOVAES, J.S. Respostas cardiovasculares e metabólicas do step training em diferentes alturas de plataforma. Rev. Bras. de Atividade Física e Saúde., v.15, n.7, p. 5-13, 2002.
MATHEUS, L.M.; MAZZARI, C.F.; MESQUITA, R.A.O. Influência dos exercícios perineais e dos cones vaginais, associados à correção postural, no tratamento da incontinência urinária feminina. Rev.

Bras. Fisioter., v.10, n.4, p. 27-39, 2006.

OLSON, M.S.; WILLIFORD, H.N.; BLESSING, D. The cardiovascular and metabolic effects of ench stepping exercise in females. Medicine and Science in Sports and Exercise., v.23, n.12, p. 1311-1317, 1991.

ORTIZ, O.C.; NUNEZ, F.C.; IBANEZ, G. Evaluación funcional del piso pelviano feminino (clasificación funcional). Bol. Soc. Latinoameric Uroginecol Cir. Vaginal., v.3, n.1, p. 5-9, 1996.

RACCO, R. O livro de ouro do pompoarismo. São Paulo: Superpedido, 2006.

RETT, M.T. Qualidade de vida em mulheres após tratamento da incontinência urinária de esforço com fisioterapia. Rev. Bras. Ginecol. Obstet., v.29, n.3, p. 134-145, 2007.

SILVA, C.R. Cinesioterapia do assoalho pélvico feminino abordagem fisioterapeutica na incontinência urinária e nas disfunções sexuais femininas. São Paulo: Phorte, 2011.

SIMÃO, R. Fisiologia e prescrição de exercícios para grupos especiais. São Paulo: Phorte, 2004. 